\title{
Therapeutics
}

\section{Review: glucocorticoids improve symptoms of croup within 6 hours}

\author{
Ausejo M, Saenz A, Pham B, et al. The effectiveness of glucocorticoids in treating croup: meta-analysis. BMJ 1999 Sep \\ 4;319:595-600.
}

QUESTION: In children with croup, what is the effectiveness of glucocorticoids on croup severity, length of hospital stay, and need for additional interventions?

\section{Data sources}

Studies were identified by searching Medline (1966 to August 1997), EMBASE/Excerpta Medica (1974 to August 1997), and the Cochrane Library Controlled Trials Register. Authors of trials published in the past 5 years were contacted.

\section{Study selection}

Studies were selected if they were randomised controlled trials of glucocorticoids compared with placebo or another active treatment in patients with croup and if clinically relevant outcome measures were used (clinical score, length of hospital stay, or need for additional interventions).

\section{Data extraction}

Data were extracted on patient characteristics, details about the intervention and control, and outcomes. The main outcome measure was improvement from baseline croup score. Additional outcomes were length of stay in the hospital or emergency department, rate of hospitalisation, and use of additional interventions.

\section{Main results}

24 studies were included; 19 were placebo controlled. Age ranged from 4 months to 12 years (mean range 13 to $45 \mathrm{mo}$ ). 17 trials evaluated dexamethasone, 9 evaluated budesonide, and 3 evaluated methylprednisolone. Improvement in croup score was measured at 6 hours in 13 studies, 12 hours in 7 studies, and 24 hours in 5 studies. The pooled effect sizes (ESs) showed an improvement in croup score with glucocorticoids at 6 hours (pooled ES 1.0, 95\% CI 0.6 to 1.5) and 12 hours (pooled ES 1.0, CI 0.4 to 1.6). At 24 hours the improvement in croup score did not reach statistical significance (pooled ES 1.0, CI -0.1 to 2.0). When children were assessed as clinically improved or not, glucocorticoids showed a benefit at all 3 time points (table). The use of adrenaline as an additional intervention decreased in children who received glucocorticoids (absolute decrease of $9 \%$ in budesonide recipients and 12\% in dexamethasone recipients [number needed to treat of 10]). Additional use of antibiotics or supplemental glucocorticoids did not increase. Children who received glucocorticoids spent less time in the emergency department (weighted mean decrease $11 \mathrm{~h}, \mathrm{CI} 4$ to 18 ), and inpatients spent less time in the hospital (weighted mean decrease $16 \mathrm{~h}$, CI 1 to 31). The use of glucocorticoids did not affect the rate of hospitalisation.

\section{Conclusion}

In children with croup, glucocorticoids relieve symptoms of croup within 6 hours of treatment.

\section{COMMENTARY}

The review by Ausejo and colleagues shows glucocorticoids to be superior to placebo in ameliorating symptoms of croup at 6 and 12 (but not 24) hours after administration and in diminishing the need for inhaled adrenaline. The effect of the reviewed drugs might also apply to other preparations (eg, prednisone suppositories, which are used in central Europe).

The review used meta-analysis to examine the effectiveness of glucocorticoids. The individual clinical relevance depends on the baseline severity, which was not given for the pooled data. It can be shown, however, from a typical study: ${ }^{2}$ croup patients at baseline had a mean of 3.8 points on the Westley croup score, and after 5 hours the 2 intervention groups had decreases of 2.0 to 2.9 points compared with a 1.3 point decrease in the placebo group. The children in the studies in this meta-analysis were moderately ill. They improved in the croup specific score, but a greater benefit was seen when they were assessed for clinical improvement.

Nebulised budesonide and oral and parenteral dexamethasone (less expensive with no difference in efficacy) can be recommended for acute croup in children. Close monitoring is necessary during the first day of glucocorticoid treatment, however, because such treatment does not substantially lower the hospitalisation rate.

Croup occasionally affects 1 child several times and might occur on a hereditary basis. ${ }^{3}$ Thus, the early parental administration of glucocorticoids to children with recurrent croup or to their siblings having a first episode should be evaluated to see whether it lowers the need for emergency visits or hospitalisation.

Johannes Forster, MD St Josefskrankenhaus Freiburg, Germany

1 Zach MS, Modl M. Monatsschr Kinderheilkd 1998;146:914-23

2 Johnson DW, Jacobson S, Edney PC, et al. N Engl J Med 1998;339:498-503.

3 Camilla AE, Holberg CJ, Wright AL, et al. Pediatr Pulmonol 1993;16:275-80
Source of funding: in part, Health Research Fund from the government of Spain.

For correspondence: Dr T P Klassen Department of Pediatrics, University of Alberta, 2C3.67 Walter C. Mackenzie Health Sciences Center,

Edmonton, Alberta T6G 2R7, Canada. Fax +1780407 8538 .

A modified version of the abstract also appears in Evidence Based Nursing

Glucocorticoids v placebo for clinical improvement in children with croup*

\begin{tabular}{|c|c|c|c|c|c|}
\hline \multirow[b]{2}{*}{ Time point } & \multirow{2}{*}{$\begin{array}{l}\text { Number of } \\
\text { studies }\end{array}$} & \multicolumn{2}{|c|}{ Weighted event rates } & \multirow[b]{2}{*}{ RBI (95\% Cl) } & \multirow[b]{2}{*}{ NNT (CI) } \\
\hline & & Glucocorticoids & Placebo & & \\
\hline $6 \mathrm{~h}$ & 13 & $56 \%$ & $41 \%$ & $37 \%$ (6 to 56 ) & 7 (4 to 50$)$ \\
\hline $12 \mathrm{~h}$ & 7 & $89 \%$ & $68 \%$ & $31 \%$ (16 to 43 ) & 5 (3 to 11$)$ \\
\hline $24 \mathrm{~h}$ & 5 & $95 \%$ & $83 \%$ & $14 \%$ (4 to 24 ) & 8 (5 to 33$)$ \\
\hline
\end{tabular}

*Abbreviations defined in glossary; $\mathrm{RBI}, \mathrm{NNT}$, and $\mathrm{Cl}$ calculated from data in article. 